\title{
Real-Space Measurement of Surface Roughening
}

\author{
M. S. Hoogeman, ${ }^{1, *}$ M. A. J. Klik, ${ }^{1}$ D. C. Schlößer, ${ }^{1, \dagger}$ L. Kuipers, ${ }^{1,+}$ and J. W. M. Frenken ${ }^{2,1}$ \\ ${ }^{1}$ FOM-Institute for Atomic and Molecular Physics, Kruislaan 407, 1098 SJ Amsterdam, The Netherlands \\ ${ }^{2}$ Kamerlingh Onnes Laboratory, Leiden University, P.O. Box 9504, 2300 RA Leiden, The Netherlands
}

(Received 29 April 1998)

\begin{abstract}
We use variable-temperature scanning tunneling microscopy (STM) combined with pattern recognition of STM images to demonstrate that the vicinal $\operatorname{Ag}(115)$ surface undergoes a true KosterlitzThouless(KT)-type roughening transition. As predicted, the height correlation function remains finite below the roughening temperature and it evolves to a logarithmically diverging function at and above $T_{R}$. From a quantitative analysis of the height fluctuations we derive a roughening temperature of $T_{R}=465 \pm 25 \mathrm{~K}$. Recent diffraction experiments could not confirm a KT-type roughening transition on vicinal surfaces. We attribute this to small residual errors in local surface orientation, which overshadow the real thermally excited height fluctuations. [S0031-9007(99)08538-5]
\end{abstract}

PACS numbers: 68.35.Rh, 07.79.Cz

Since the first prediction by Burton, Cabrera, and Frank [1] that flat single-crystal surfaces may become rough above a certain temperature, $T_{R}$, this so-called roughening transition has been the subject of much theoretical and experimental work $[2,3]$. The thermal roughening influences surfaces on all length scales. On the microscopic scale, atomic steps proliferate, which causes height fluctuations that make the flat faces disappear from the macroscopic equilibrium crystal shape. The dramatic change of the surface morphology can strongly influence various properties, such as the growth behavior and the chemical reactivity of a crystal surface $[1,4]$.

Most experimental work on roughening has been performed with diffraction techniques, which probe the Fourier transform of the height correlation function, $G(\rho)=\left\langle\left(h_{i}-h_{j}\right)^{2}\right\rangle$, where $h_{i}$ is the height at site $i$, $\rho$ is the distance between sites $i$ and $j$, and the brackets denote the ensemble average. Statistical mechanical models predict a Kosterlitz-Thouless(KT)-type transition [5]. At temperatures below $T_{R}, G(\rho)$ should remain finite for all $\rho$, while it should diverge logarithmically with $\rho$ at and above the transition temperature. Although an impressive body of work with $\mathrm{He}$ atom and $\mathrm{x}$-ray diffraction from vicinal $\mathrm{Cu}$ and $\mathrm{Ni}$ surfaces originally seemed to be in full agreement with these predictions [2], more refined $\mathrm{He}$ and electron diffraction measurements $[6,7]$ have recently disqualified the original results. After a proper energy analysis, the diffraction profiles do not have the expected shape and dependence on temperature. Also in experiments using real-space techniques $G(\rho)$ is found to deviate strongly from the theoretical prediction [8-10].

In this Letter we present the direct measurement of the height correlation function on the vicinal $\operatorname{Ag}(115)$ surface, with high-speed, variable-temperature scanning tunneling microscopy (STM). We show that at no temperature $G(\rho)$ approaches a constant value or increases logarithmically with $\rho$, and we find that the origin of this behavior lies in residual errors in local surface orientation. The resulting height variations completely overshadow the more subtle contribution of the thermal roughening transition to the surface roughness. When these "nonthermodynamic" height variations are removed from the height correlation function, we obtain a correlation function that exhibits the expected shape and dependence on temperature, thus providing the first model-independent proof of a KT-type transition.

In the following we briefly introduce the experimental procedure and explain how the measurements are corrected for the effects of errors in local surface orientation. The results are almost completely in quantitative agreement with the prediction of the Terrace-Ledge-Kink (TLK) model of Villain et al. [11].

The perfect (115) surface consists of (001) microterraces with a width of 2.5 atomic spacings, separated by (111)type steps (Fig. 1). Height differences are caused by the narrowing or broadening of the (001) microterraces. The energy penalty for the local displacement of a step is twice the kink formation energy plus the increase of the step interaction energy along the displaced step segment. A collective displacement of several steps results in an island on the (115) terrace. Thermally excited step displacements are responsible for the thermal roughening of the (115) facet. According to the TLK model, the magnitudes of the kink formation and step interaction energies determine the roughening temperature [11]. For $\operatorname{Ag}(115)$ these energies are known from a previous STM study [12], and the TLK model predicts that $T_{R}=427 \pm 10 \mathrm{~K}$.

The $\operatorname{Ag}(115)$ sample $\left(10 \times 10 \times 3 \mathrm{~mm}^{3}\right)$ was spark cut from a high-purity $(6 N)$ single-crystal ingot. The crystal was chemically etched, oriented to within $0.05^{\circ}$ of the (115) plane by means of Laue x-ray diffraction, and mechanically polished in this orientation. After this it was transferred to the scanning tunneling microscope. The surface was cleaned in situ by cycles of Ar ion sputtering at $800 \mathrm{~K}$ and annealing to $900 \mathrm{~K}$, followed by slow cooling. The level of impurities was below the $1 \%$ detection limit of Auger electron spectroscopy. After a few tens of cycles the maximum terrace width was $400 \AA$, although 


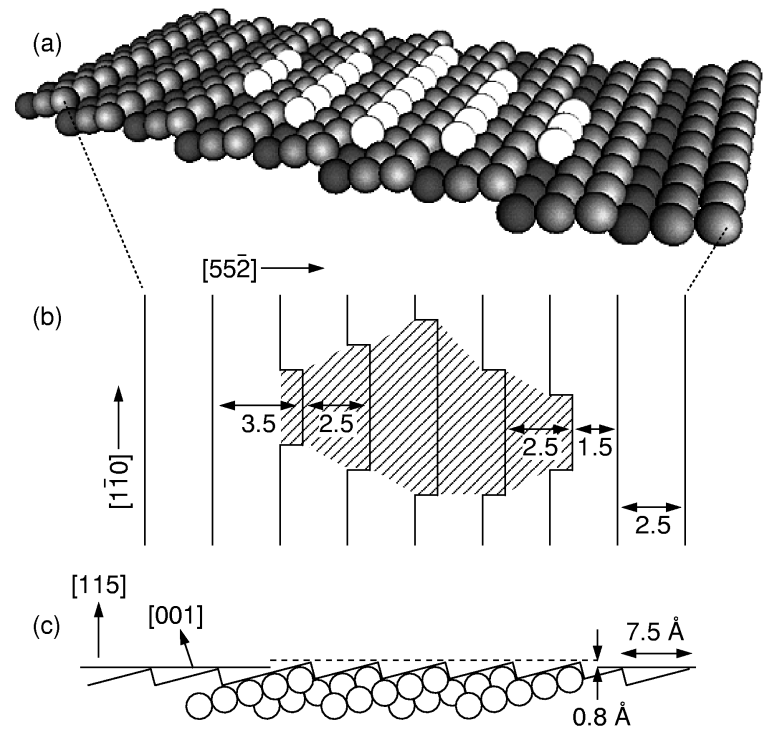

FIG. 1. Perspective (a), top (b), and side (c) views of the $\operatorname{Ag}(115)$ surface. Nine (111)-type steps are shown. The collective displacement of the steps results in an island on the (115) terrace. Thermally excited height fluctuations like these are responsible for the roughening of this surface. The numbers in (b) indicate the widths of the (001) microterraces in atomic spacings.

regions occurred with significantly narrower terraces. The experiments were performed with a high-speed, variabletemperature STM under ultrahigh vacuum $(p<2 \times$ $10^{-10}$ mbar) [13]. The STM data were obtained with tunneling currents in the range of 0.05 to $0.3 \mathrm{nA}$ and sample bias voltages ranging from +150 to $+250 \mathrm{mV}$. The imaging time per frame varied from 1.3 to $8 \mathrm{~s}$ and the scan lines (fast direction) were oriented along the [55̄] direction, perpendicular to the steps.

Figure 2 shows a STM image of $\operatorname{Ag}(115)$ at $425 \mathrm{~K}$. Three "steps" running across the entire image separate the (115) terraces. The grid of lines on the (115) terraces reflects the lattice of (111)-type steps with a period of $7.5 \AA$. The "noise" on each of the terraces originates from spontaneous local height fluctuations. These are the fluctuations that eventually lead to the roughening of $\operatorname{Ag}(115)$ at higher temperatures. Each of the fluctuations in Fig. 2 corresponds to a well-defined displacement of one or several of the (111)-type steps over a small number of atomic spacings, usually just one. The three steps indicated by the arrows are due to narrowed (001) terraces (see Fig. 1) and accommodate a modest $0.5^{\circ}$ local misorientation of the surface. On a larger scale the surface was not perfectly flat, and its orientation varied around (115), with a (temperature-independent) root-mean-square deviation of $0.20^{\circ}$ and with wavelengths of $2000 \AA$ and larger. This corresponds to a waviness amplitude below $2 \AA$. Even with careful chemical or mechanical polishing, metal surfaces cannot be made perfectly flat. Although the waviness amplitude had reduced significantly in the course of extended sputter/anneal cycles, it was not possible to ob-

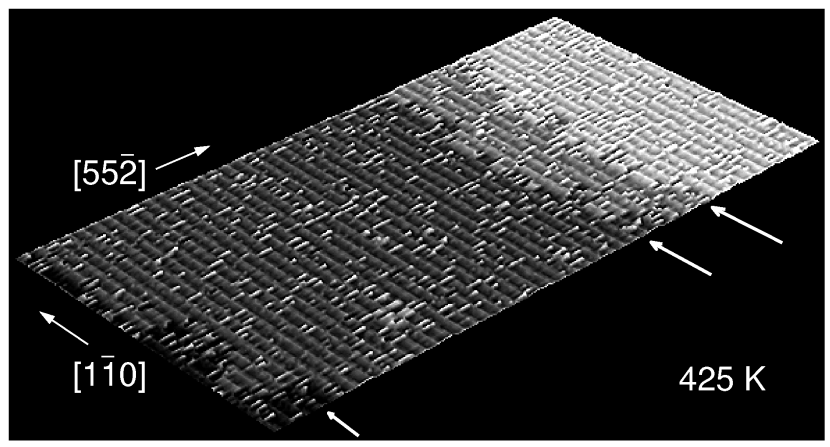

FIG. 2. Perspective representation of a STM image, measured at $425 \mathrm{~K}\left(330 \times 165 \AA, V_{t}=250 \mathrm{mV}, I_{t}=0.1 \mathrm{nA}\right)$. The (111)-type steps are clearly resolved as well as three steps which separate the (115) terraces, indicated by arrows (see text). The noise on each of the terraces originates from spontaneous local height fluctuations.

tain the large terrace widths of several thousand angstrom, which we routinely find on low-index surfaces. It is the combination of the low energetic driving force for the flattening of a vicinal surface, the modest surface mobility at temperatures below $T_{R}$, at which the smoothing should really occur, and the relatively small step height on $\operatorname{Ag}(115)$ of only $0.8 \AA$ that makes the time needed for the growth of (115) terraces beyond $400 \AA$ excessively long.

We have determined $G(\rho)$ along the fast [552̄] direction from images such as Fig. 2. The natural corrugation of the step grid on the (115) terraces leads to pronounced oscillations in $G(\rho)$ that overshadow the contribution from the surface roughness. Therefore, we discretized the step positions detected in each scan line in terms of the number of unit displacements of each step with respect to a grid of reference positions. $G(\rho)$ is then calculated as the height or displacement correlation function between the integer step position values. Whereas it is easy to perform the discretizing by hand in low-temperature images such as Fig. 2, an unambiguous assignment by hand is very difficult at higher temperatures, where the fluctuations are much more abundant and have larger amplitudes. Therefore, we have developed a pattern recognition procedure, which automatically finds the step positions and corresponding heights. The procedure uses the crystallographic information of the possible step heights and step distances on $\operatorname{Ag}(115)$. The algorithm is insensitive to statistical noise in the image and is extremely robust with respect to bad resolution of the STM tip and even resolution changes within an image. Thermal drift and piezocreep are all recognized and do not affect the identification of the step positions. A preliminary description of the procedure can be found in Ref. [14]. A full account will be given in a future publication.

From the integer values of the surface height, obtained from the pattern recognition, we calculate directly the height correlation function $G(\rho)$. Figure 3a shows the result for four different temperatures around the expected roughening temperature [12]. Typically 50 images of 


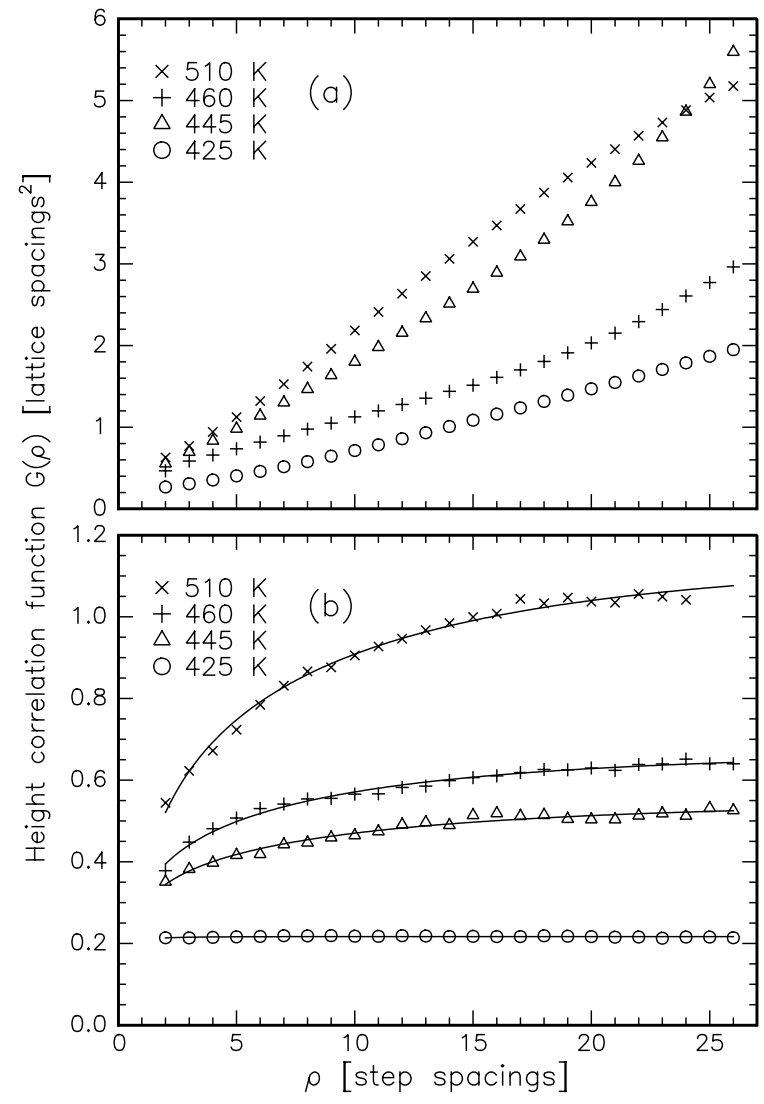

FIG. 3. Height correlation function measured along [552̄] at different temperatures. (a) $G(\rho)$ measured directly from the discretized step positions. The height correlation function is dominated by residual surface orientation errors. (b) $G(\rho)$ after correction for local orientation errors. The measured data follow the behavior expected for the roughening transition. The solid curves are fits to the data according to Eq. (1).

different surface configurations were used per temperature, adding up to a total probed area of $\approx 1.5 \times 10^{7} \AA^{2}$.

Around $T_{R}, G(\rho)$ is expected to behave as $[2,11,15]$

$$
G(\rho)=-K(T) \ln \left[\rho^{-2}+\kappa^{2}(T)\right]+C(T),
$$

where the inverse correlation length $\kappa(T)$ is zero for $T \geq T_{R}$ and nonzero for $T<T_{R}$. The prefactor $K(T)$ is predicted to reach the universal value $1 / \pi^{2}$ at $T_{R}$ after which it continues to increase with temperature. The ratio $C(T) / 2 K(T)$ has recently been predicted to become equal to 2 for $T>T_{R}$ [10].

At none of the temperatures in Fig. 3a can the measured height fluctuations be described by Eq. (1). Instead, $G(\rho)$ seems to increase more or less linearly at each temperature, in accordance with the recent diffraction results in Refs. [6,7]. Furthermore, the height correlation function does not rise monotonically with temperature. We will now show that the residual variation in surface orientation completely dominates the measured height correlation function. As discussed above, height changes as indicated by the arrows in Fig. 2 form part of a mesoscopic scale waviness which is not completely removed by the surface preparation and is not related to the less pronounced, temperature-dependent roughness involved in the roughening transition. In Fig. 2 the modest $0.5^{\circ}$ misorientation is responsible for more than $75 \%$ of the $G(\rho)$ value for the higher $\rho$ 's.

We minimize the contribution from surface orientation errors by automatically selecting small regions in the images with the proper average orientation. The idea behind this method is that, locally, within such a region, the height changes are not "necessary" to accommodate any misorientation, so that they may be regarded as truly thermally excited height fluctuations. We apply this selection by placing a one-dimensional window over each scan line and accepting only those window positions where the scan line has the same height at the beginning and at the end of the window. The width of the window is smaller than that of the image, so that there are many candidate window positions per scan line.

The finite size effects produced by the selection procedure are minute for $\rho$ 's smaller than half the window width. The equal-height condition at the window's ends is very similar to periodic boundary conditions in computer simulations. In several publications, finite-size effects in Monte Carlo (MC) simulations of the roughening transition have been discussed $[15,16]$. For cell widths smaller than 32 units, Mazzeo et al. have measured a deviation of $T_{R}$ of only $2 \%$ from the analytical prediction [16]. To obtain further confidence in our selection procedure, we performed $\mathrm{MC}$ simulations of the roughening transition as well [17]. We measured in a large simulation cell the height correlation function, for which we applied the same selection criterion as described above, for several window widths between 20 and 61 step spacings. The simulations showed that for these window widths the measured deviation of $T_{R}$ is less than $2 \%$ from the prediction of the TLK model.

Figure $3 \mathrm{~b}$ shows the height correlation function obtained from the same data as in Fig. 3a but corrected for local orientation errors by the selection method described above for a window width of 34 step spacings. The difference between Figs. $3 a$ and $3 b$ is dramatic. Removing the effect of the orientation errors has reduced $G(\rho)$ by more than a factor of 5. The shape of the correlation functions has changed completely and a clear development of $G(\rho)$ with temperature has emerged. As predicted, the correlation function quickly converges to a constant value at low temperatures, whereas it increases logarithmically at the higher temperatures.

The solid curves are fits to the data according to Eq. (1). Figure 4 shows $K(T)$ and $\kappa(T)$. The prefactor crosses the universal value of $1 / \pi^{2}$ between 460 and $510 \mathrm{~K}$. Between 425 and $445 \mathrm{~K}, \kappa(T)$ drops to a value close to 0 . In the same temperature regime $C(T) / 2 K(T)$ approaches 2 [18].

$K(T)$ crosses $1 / \pi^{2}$ at a slightly higher temperature than that at which $\kappa(T)$ drops to 0 . The reason for this is not yet clear. The MC simulations do not show such a difference in temperature so that we know that it is not a finite-size effect. The small deviation of $\kappa(T)$ from 0 at the higher temperatures is a finite-size effect and will 


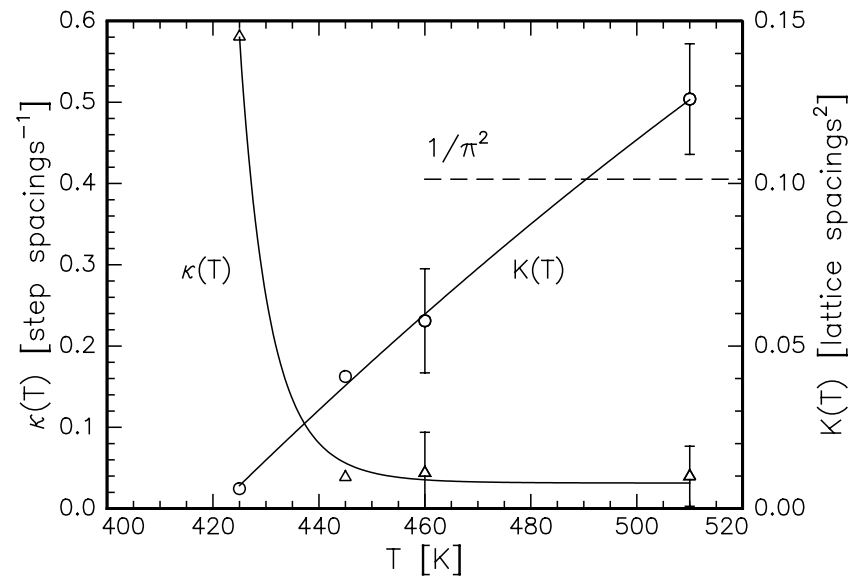

FIG. 4. Prefactor $K(T)$ and inverse correlation length $\kappa(T)$ as a function of temperature. The solid curves serve to guide the eye. $K(T)$ crosses the universal value of $1 / \pi^{2}$ between 460 and $510 \mathrm{~K}$, while $\kappa(T)$ approaches its minimum value between 425 and $445 \mathrm{~K}$.

be discussed in more detail in a future publication [17]. From the data in Fig. 4 we conclude that $\mathrm{Ag}(115)$ roughens at $T_{R}=465 \pm 25 \mathrm{~K}$. This value is consistent with an earlier, lower estimate of $T_{R}$ of $450 \mathrm{~K}$, which was based on the qualitative comparison between STM images [12].

In an earlier publication [12] we used the TLK model [11] to predict a transition temperature of $427 \pm 10 \mathrm{~K}$. Input in this prediction were accurate values for the step interaction energy, $w_{2}=38 \pm 4 \mathrm{~K}$, and for the kink creation energy, $W_{0}=1323 \pm 10 \mathrm{~K}$, determined from step and kink statistics in STM movies on $\operatorname{Ag}(115)$ at room temperature. The MC simulations are consistent with the prediction of the TLK model and will be presented in a future publication [17]. The experimental value of $T_{R}=465 \pm 25 \mathrm{~K}$ determined from Fig. 4 is close to the predicted value, which is remarkable in view of the relative simplicity of the TLK model.

We note that one cannot identify any length scale in Fig. 3a on which the effect of the small orientation errors can be neglected. Since the distance range in Fig. 3 corresponds to the typical reciprocal space range probed in a surface diffraction experiment, there is no straightforward manner in which the effect of the orientation variations can be removed from the diffraction line shapes. The correction method employed here seems possible exclusively for real-space data.

In summary, we have used a scanning tunneling microscope to demonstrate that the $\operatorname{Ag}(115)$ surface undergoes a Kosterlitz-Thouless-type roughening transition at $T_{R}=465 \pm 25 \mathrm{~K}$. As predicted theoretically, the height correlation function $G(\rho)$ evolves from having a finite asymptotic value at temperatures below $T_{R}$ to displaying a logarithmic divergence above the transition. The proper form and temperature dependence of $G(\rho)$ are observed only if the raw STM data are corrected for the effect of the residual surface orientation errors remaining after the surface preparation.
The authors gratefully thank L. Barbier for helpful discussions. The authors acknowledge R. J.I. M. Koper for the preparation of the $\operatorname{Ag}(115)$ sample and $\mathrm{E}$. Vlieg for a critical reading of the manuscript. This work is part of the research program of the Foundation for Fundamental Research on Matter (FOM) and was made possible by financial support from the Netherlands Organization for the Advancement of Research (NWO).

*Present address: The Netherlands Cancer Institute/Antoni van Leeuwenhoek Huis, Plesmanlaan 121, 1066 CX Amsterdam, The Netherlands.

†Present address: P\&G European Service GmbH, H.-S.Richardson-Strasse, 64521, Gross-Gerau, Germany.

*Present address: Twente University, Department of Applied Physics, P.O. Box 217, 7500 AE Enschede, The Netherlands.

[1] W. K. Burton, N. Cabrera, and F. C. Frank, Philos. Trans. R. Soc. London A 243, 299 (1951).

[2] J. Lapujoulade, Surf. Sci. Rep. 20, 191 (1994), and references therein.

[3] H. van Beijeren and I. Nolden, in Structure and Dynamics of Surfaces II, edited by W. Schommers and P. von Blanckenhagen (Springer, Berlin, 1987), p. 259.

[4] G. Beitel, C.P. M. de Groot, H. Oosterbeek, and J.H. Wilson, J. Phys. Chem. B 101, 4035 (1997).

[5] S. T. Chui and J. D. Weeks, Phys. Rev. B 14, 4978 (1976).

[6] J. Wollschläger, E.Z. Luo, and M. Henzler, Phys. Rev. B 44, 13031 (1991).

[7] H.-J. Ernst, R. Folkerts, and L. Schwenger, Phys. Rev. B 52, 8461 (1995).

[8] P.E. Hegeman, H. J. W. Zandvliet, G. A. M. Kip, B. A. G. Kersten, and B. Poelsema, Surf. Sci. 331-333, 1110 (1995).

[9] J. C. Heyraud, J.M. Bermond, C. Alfonso, and J. J. Métois, J. Phys. I (France) 5, 443 (1995).

[10] L. Barbier, L. Masson, J. Cousty, and B. Salanon, Surf. Sci. 345, 197 (1996).

[11] J. Villain, D. R. Grempel, and J. Lapujoulade, J. Phys. F 15, 809 (1985).

[12] M. S. Hoogeman, D. C. Schlößer, J. B. Sanders, L. Kuipers, and J.W.M. Frenken, Phys. Rev. B 53, R13 299 (1996).

[13] L. Kuipers, R. W. M. Loos, H. Neerings, J. ter Horst, G. J. Ruwiel, A.P. de Jongh, and J. W. M. Frenken, Rev. Sci. Instrum. 66, 4557 (1995).

[14] J. W. M. Frenken, L. Kuipers, and J. B. Sanders, Faraday Discuss. 95, 27 (1993).

[15] W. Selke and A. M. Szpilka, Z. Phys. B 62, 381 (1986).

[16] G. Mazzeo, G. Jug, C. Levi, and E. Tosatti, Surf. Sci. 273, 237 (1992).

[17] M. S. Hoogeman and J. W. M. Frenken (unpublished).

[18] For $T>T_{R}$ the value of $C(T) / 2 K(T)$ should approach 2 , as predicted by Barbier et al. [10] for $\rho$ taken perpendicular to the steps. The prediction follows from the sine-Gordon approach to calculate the height-correlation function. Whether this approach is also valid for the lowindex vicinal surfaces such as the [113] and [115] orientations is not yet clear. 